\title{
ESTIMACIÓN Y PREDICCIÓN DE LAS PROPIEDADES MECÁNICAS En Pinus radiata D. Don DE 30 AÑOS DE EDAD MEDIANTE REGRESIÓN MÚLTIPLE
}

\author{
Carlos Rozas*, Gilda Vargas Mc**, José Anzaldo H*** \\ *M.Sc., Doctorando en Ing. Forestal, UFPR, Universidad del Bío - Bío, Concepción, Chile - erozas@ubiobio.cl \\ **M.Sc., Universidad del Bío - Bío, Concepción, Chile - gvargas@ubiobio.cl \\ ***M.Sc., Universidad de Guadalajara, Guadalajara, México - janzalo@cucei.udg.mx \\ Recebido para publicação: 10/07/2006 - Aceito para publicação: 22/09/2006
}

\begin{abstract}
Resumen
Este estudio presenta los resultados del Proyecto FONDEF D97I2006 desarrollado en la Universidad del Bío-Bío (Chile). El objetivo del estudio fue desarrollar modelos de regresión múltiple para estimar las propiedades mecánicas de la madera de Pinus radiata D. Don, considerando las propiedades físicas de la madera, tipo de madera (juvenil y adulta) y diferentes zonas de crecimiento. Los modelos desarrollados permitieron obtener una buena estimativa de las propiedades mecánicas. La dureza paralela, dureza perpendicular, el MOR, el MOE y el esfuerzo máximo en compresión paralela, obtuvieron los coeficientes de determinación más altos, siendo de 0,$82 ; 0,84 ; 0,80 ; 0,70$ y 0,70 , respectivamente.

Palabras claves: Propiedades mecánicas; Pinus radiata D. Don; regresión múltiple.
\end{abstract}

\begin{abstract}
Assessment and forecasting of mechanical properties for the 30-year-old Pinus radiata D. Don BY means of the multiple regression system. This paper presents the results of FONDEF Project D9712006 developed at Bío-Bío University (Chile). The objetive of the study was to develop multiple regression models to estimate mechanical properties of Pinus radiata D. Don wood taking into account physical properpies, wood type (juvenile and adult wood) and different wood source (regions). The models developed allow to obtain good estimates of the mechanical propierties. Parallel hardness, perpendicular hardness, MOR, MOE and maximum stress in parallel compression presented the highest determining coefficients, being $0.82 ; 0.84 ; 0.80 ; 0.70$ and 0.70 , respectively. Keywords: Mechanical properties; Pinus radiata D. Don; multiple regression.
\end{abstract}

\section{INTRODUCCIÓN}

Una de las principales especies que crecen en Chile es el Pinus radiata D. Don, que fue introducida en este país hacia fines del siglo XIX, viniendo incluida por descuido entre otras semillas de coníferas (DÍAZ, 1985). Debido a su rápida climatización y crecimiento, hoy se extiende entre la V y X región del país, alcanzando en estas zonas los mayores niveles de plantación (PASTÉN; RUBILAR, 2001).

En Chile, los bosques plantados actualmente de Pinus radiata D. Don, alcanzan una superficie aproximada de 1.458 .320 hectáreas, equivalente a un $74,7 \%$ del total de plantaciones, esperándose una disponibilidad para el trienio 2001-2003 de 19,6 millones de $\mathrm{m}^{3}$, de los cuales 10,6 millones de $\mathrm{m}^{3}$ se destinarían a madera aserrada (INFOR, 1999).

Este estudio surge de la necesidad de encontrar una forma más práctica de caracterizar la madera de Pinus radiata D. Don, por un método no destructivo, es así que al determinar la densidad de un árbol conociendo su edad y procedencia, se pueda estimar las propiedades mecánicas de éste. Esto porque las propiedades mecánicas de la madera son afectadas por varios factores, principalmente por la densidad, el contenido de humedad, la temperatura, duración de la carga, inclinación de la fibra, entre otros (BODIG; JAYNE, 1993). Diversos autores han correlacionado las propiedades mecánicas con la densidad, es más, 
se llegó a establecer una ecuación general donde se establece matemáticamente la relación entre la densidad y cualquier propiedad mecánica (KARSULOVIC, 1982; BODIG; JAYNE, 1993).

Un estudio similar en el cual se basó el Proyecto FONDEF D97I2006, se realizó en Nueva Zelanda por Kininmonth; Whitehouse (1991), quienes estudiaron algunas propiedades y usos de la madera de Pinus radiata D. Don proveniente de Nueva Zelanda. Analizando entre otros el efecto de la densidad, características de crecimiento, ancho de anillo, contenido de humedad y condiciones de los ensayos mecánicos de la madera.

Jopia (2000), realizó un análisis factorial con el fin de reducir las dimensiones de un gran número de variables observadas y poder caracterizar las propiedades mecánicas del Pinus radiata D. Don, proveniente de una de las zonas que también contempló este estudio (Arenales Bulnes - Mulchén); se concluyó que para caracterizar la madera se debe tener en cuenta un factor relacionado con las propiedades mecánicas, densidad y tipo de madera, y otro relacionado con la edad.

Juacida et al. (2000), realizó un estudio similar con Populus x euroamericana (Dode) Guinier, quien determinó las propiedades mecánicas de tensión en el límite proporcional, MOR y MOE en flexión; tensión máxima en compresión paralela y perpendicular a las fibras; tensión máxima en tracción normal y cizalle paralelo a las fibras; clivaje y dureza Janka, las que correlacionó con la densidad, concluyendo que las propiedades que más se relacionaron con la densidad de la madera fueron el MOR y las tensiones máximas compresión paralela y perpendicular a las fibras.

En la octava región (Chile), se realizaron dos estudios similares, el primero lo realizó Albala (1964). En ese estudio, se subdividió el área de procedencia de la madera en dos zonas, donde trabajaron con 10 árboles en cada una. Se realizaron ensayos a un $12 \%$ de contenido de humedad y en estado verde, obteniéndose similar tendencia en los resultados de ambas zonas. El segundo estudio fue realizado por Pérez; Cabrera (1987), quienes estudiaron la incidencia de la edad del árbol sobre las propiedades mecánicas, compararon los valores medios de las propiedades mecánicas de árboles de 25 y 35 años provenientes de Arauco, a un $12 \%$ de contenido de humedad y en estado verde, obteniéndose como resultado, que los árboles de mayor edad poseen un incremento en la resistencia promedio de $15 \%$, en comparación con los de menor edad para un $12 \%$ de contenido de humedad.

Actualmente, también se cuentan con estudios realizados sobre determinación de las propiedades mecánicas dentro del marco del Proyecto FONDEF D97I2006, para la zona costera de Constitución Cobquecura (PASTÉN; RUBILAR, 2001), zona de arenales Bulnes - Mulchén (GALLARDO, 1999) y zona de pre-cordillera de los Andes para árboles de 20 años de edad (PEREIRA, 2000). A ello se suman los Informes técnicos realizados por Rozas; Pastén (2001), como parte de los resultados del Proyecto FONDEF D97I2006.

De acuerdo a los antecedentes anteriores, un acabado conocimiento de las propiedades mecánicas de la madera, permite determinar cuales son los factores críticos que las determinan, estableciendo las condiciones adecuadas, como zona geográfica de procedencia y edad, para obtener una materia prima más competitiva y con mayor valor agregado, principalmente para fines estructurales (ROZAS; PASTÉN, 2001).

El objetivo de este estudio fue estimar y predecir las propiedades mecánicas de la madera de Pinus radiata D.Don de 30 años de edad mediante regresión múltiple, utilizándose para ello la base de datos del Proyecto FONDEF D97I2006 "Caracterización de la madera de Pinus radiata D. Don, para la VII, VIII y IX región", donde se relacionaron las propiedades mecánicas con la edad, densidad, zona de crecimiento y tipo de madera.

\section{MATERIAL Y MÉTODOS}

\section{Procedencia de los árboles seleccionados}

Fueron seleccionados un total de 133 árboles de 30 años de edad (Tabla 1), los cuáles provinieron de la costa Constitución - Cobquecura (zona I); Concepción - Angol, sur río Bío - Bío (zona II); arenales Bulnes - Mulchén (zona III) y Arauco - río Imperial (zona IV).

\section{Obtención de probetas}

Se obtuvieron probetas para la realización de los ensayos mecánicos (AMERICAN SOCIETY FOR TESTING AND MATERIALS, 1981 y INSTITUTO NACIONAL DE NORMALIZACIÓN - INN, 
1984a., 1984b, 1986a, 1986b, 1986c, 1986d, 1986e, 1986f, 1986g), de tracción perpendicular a las fibras (radial y tangencial), cizalle paralelo a las fibras (radial y tangencial), dureza Janka, flexión estática y compresión paralela a las fibras, en madera juvenil y adulta, a partir de viguetas de dimensiones finales $0,05 \times 0,05 \times 1,7 \mathrm{~m}$, obtenidas cercanas a la médula y a la corteza, respectivamente. Las viguetas provinieron de un tablón central que se obtuvo en cada uno de los dos trozos cortados de cada árbol, como se muestra en la figura 1 y según la norma INN, 1986a. El primer trozo corresponde a la sección del árbol entre el DAP y el $25 \%$ de la altura comercial y el segundo trozo correspondiente a la sección entre el $25 \%$ y $50 \%$ de la altura comercial, ambos trozos de $1,7 \mathrm{~m}$ de longitud.

Tabla 1. Procedencia de los árboles seleccionados de Pinus radiata D. Don de 30 años de edad.

Table 1. Procedence of Pinus radiata D. Don 30 year-old selected trees.

\begin{tabular}{|c|c|c|c|}
\hline Zona & Denominación y ubicación & Número de árboles & DAP promedio \\
\hline & Costa Constitución - Cobquecura: & & \\
\hline I & $\begin{array}{l}\text { Sur del lago Vichuquén }\left(34^{\circ} 50^{\prime} \text { latitud sur) y el río Itata }\right. \\
\left(36^{\circ} 25^{\prime} \text { latitud sur). }\right.\end{array}$ & 50 árboles & $0,39 \mathrm{~m}$ \\
\hline II & $\begin{array}{l}\text { Concepción - Angol, río Bío - Bío: } \\
\text { Sur del río Itata ( } 36^{\circ} 30^{\prime} \text { latitud sur) y sur de Traiguén } \\
\left(38^{\circ} 15^{\prime} \text { latitud sur). }\right. \\
\text { Arenales Bulnes - Mulchén: }\end{array}$ & 16 árboles & $0,22 \mathrm{~m}$ \\
\hline III & $\begin{array}{l}\text { Cabrero }\left(37^{\circ} 03^{\prime} \text { latitud sur) y el río Bío - Bío }\left(37^{\circ} 35^{\text {, }}\right.\right. \\
\text { latitud sur). }\end{array}$ & 50 árboles & $0,43 \mathrm{~m}$ \\
\hline IV & $\begin{array}{l}\text { Arauco - río Imperial: } \\
\text { Sur del río Itata ( } 36^{\circ} 30^{\prime} \text { latitud sur) y norte del río Imperial } \\
\left(38^{\circ} 45^{\prime} \text { latitud sur). }\right.\end{array}$ & 17 árboles & $0,41 \mathrm{~m}$ \\
\hline
\end{tabular}

Fuente: Proyecto FONDEF D97I2006

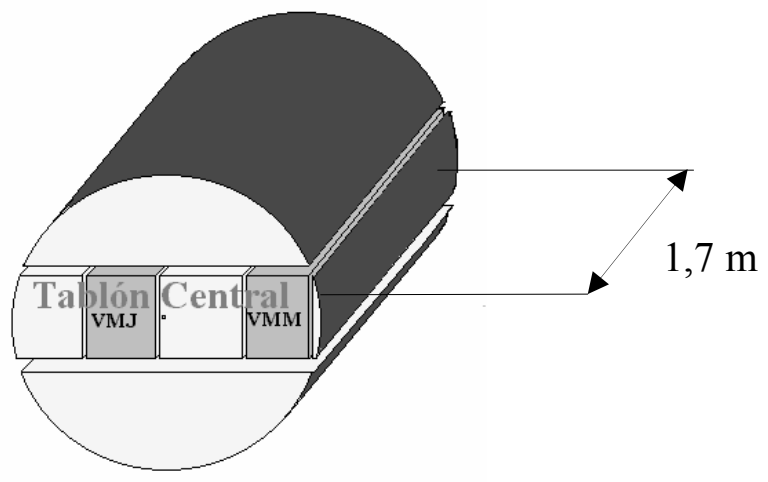

Figura 1. Esquema de corte para obtener el tablón central, la vigueta de madera juvenil (VMJ) y la vigueta de madera madura (VMM).

Figure 1. Juvenile wood lumbers and adult wood lumber test specimens obtainment.

\section{Determinación de las propiedades mecánicas}

Las probetas se acondicionaron en cámara de clima, hasta obtener un contenido de humedad de equilibrio del 12\% (INN NCh 969 of.86). Posteriormente se realizaron los ensayos mecánicos en la máquina de ensayos universales INSTRON 4468, perteneciente a la Universidad del Bío - Bío. Las propiedades determinadas fueron: tensión máxima radial $\left(\mathrm{R}_{\mathrm{tnr}}\right)$ y tangencial $\left(\mathrm{R}_{\mathrm{tnt}}\right)$ en tracción perpendicular (INN, 1986d), tensión máxima radial $\left(\mathrm{R}_{\mathrm{vr}}\right)$ y tangencial $\left(\mathrm{R}_{\mathrm{vt}}\right)$ en cizalle paralelo (INN, 1986e), dureza Janka paralela $(Q)$ y perpendicular $\left(Q_{p}\right)(I N N, 1986 f)$, MOR y MOE en flexión estática (INN, 1986g), y tensión máxima $\left(\mathrm{R}_{\mathrm{c}}\right)$ y módulo de elasticidad $\left(\mathrm{E}_{\mathrm{c}}\right)$ en compresión paralela (INN, 1986c), además se determinó a cada probeta la densidad nominal (INN, 1984b), que corresponde al cociente entre la masa al $0 \%$ de humedad y el volumen al $12 \%$ de humedad. El contenido de humedad fue determinado según INN, 1984a. 


\section{Metodología estadística}

Para la comparación entre zonas, se utilizó la prueba F bajo un diseño completamente aleatorio. El análisis entre medias se realizó mediante los tests de Duncan y Tukey. Para establecer los modelos estadísticos, se identificaron las variables dependientes o variables respuestas (propiedades mecánicas) y las independientes (zona de procedencia, tipo de madera y densidad nominal). Luego se realizó un análisis exploratorio de datos con el fin de detectar puntos atípicos y analizar el comportamiento de las variables dependientes en función de cada variable independiente. Posteriormente, se llevó a cabo el análisis de regresión y con el modelo general obtenido, se aplicó el procedimiento backward regression con un nivel de significancia del 10\%, con el fin de obtener un modelo parsimonioso, cuyo objetivo es que el modelo obtenga únicamente variables que ofrezcan una contribución definida al poder explicativo del modelo. Es así, como la no-existencia de algunas variables independientes en un modelo implica la baja influencia de éstas en la variable respuesta (JOPIA, 2000). Para los análisis estadísticos se utilizó el software estadístico SAS 6.12.

\section{RESULTADOS Y DISCUSIÓN}

\section{Madera juvenil}

En la tabla 2 se muestran los valores promedios de las propiedades mecánicas de la madera juvenil de Pinus radiata D. Don, de 30 años de edad. Los valores que no presentan diferencias estadísticas significativas están subrayados a un mismo nivel. Se observa que la gran mayoría de las propiedades mecánicas presentaron los mayores valores promedios en la zona I (costa Constitución Cobquecura), denotándose en las propiedades de tensión máxima en cizalle tangencial, dureza paralela, dureza perpendicular, MOE y MOR; seguidos de la zona II (Concepción - Angol, río Bío - Bío), en las propiedades de tensión máxima en tracción radial, tracción tangencial y tensión máxima en compresión paralela. En la zona III (arenales Bulnes - Mulchén), el módulo de elasticidad en compresión fue mayor que el resto de las zonas, y en la zona IV (Arauco - río Imperial) la tensión máxima en cizalle tangencial.

En general, las propiedades mecánicas se comportan de manera diferente al compararlas entre zonas; sin embargo, las propiedades de dureza paralela, dureza perpendicular y MOE, registraron un comportamiento similar, al igual que la tensión máxima en tracción radial y el MOE. La única propiedad en donde no existieron diferencias entre las 4 zonas fue el módulo de elasticidad en compresión paralela a las fibras; sin embargo, se evidenció que en general hubo tendencia a existir diferencias estadísticas entre zonas, pero no en forma clara.

Entre las zonas II y IV, se encontraron la mayor cantidad de propiedades mecánicas que no reflejaron diferencia estadística significativa y entre las zonas III y IV, se encontraró la mayor cantidad de propiedades que sí reflejaron diferencias estadísticas significativas.

\section{Madera adulta}

En la tabla 3 se muestran los resultados promedios de las propiedades mecánicas de la madera adulta de Pinus radiata D. Don de 30 años de edad. Se puede observar, que los mayores valores promedios de las propiedades mecánicas estimadas, se obtuvieron en la zona II (Concepción - Angol, río Bío - Bío), para las propiedades de tensión máxima en tracción radial, cizalle radial, cizalle tangencial, compresión paralela y módulo de elasticidad en compresión; seguidos de la zona I (costa entre Constitución - Cobquecura) para las propiedades de dureza paralela, dureza perpendicular y MOR, y zona IV (Arauco - río Imperial) para la tensión máxima en tracción tangencial y el MOE. En la zona III (arenales Bulnes - Mulchén), no se encontraron mayores valores promedios de propiedades mecánicas.

Las propiedades de dureza paralela y perpendicular presentaron idéntico comportamiento al compararlas estadísticamente entre zonas, al igual que en madera juvenil. También tuvieron un comportamiento similar las propiedades de tensión máxima en cizalle tangencial, compresión paralela y módulo de elasticidad en compresión. En estas tres últimas propiedades no se encontraron diferencias estadísticas significativas entre las 4 zonas.

Se observa que no existen diferencias estadísticas significativas, a diferencia de lo ocurrido en madera juvenil, principalmente, en las propiedades de tensión máxima en cizalle tangencial, compresión paralela y módulo de elasticidad en compresión; en cambio, la tensión máxima en tracción radial, fue la propiedad en donde más se reflejó la existencia de diferencias estadísticas significativas entre zonas. 
Tabla 2. Propiedades mecánicas en madera juvenil de Pinus radiata D. Don de 30 años, al 12\% de contenido de humedad.

Table 2. Mechanical properties of juvenile Pinus radiata D. Don 30 year-old wood at 12\% moisture content.

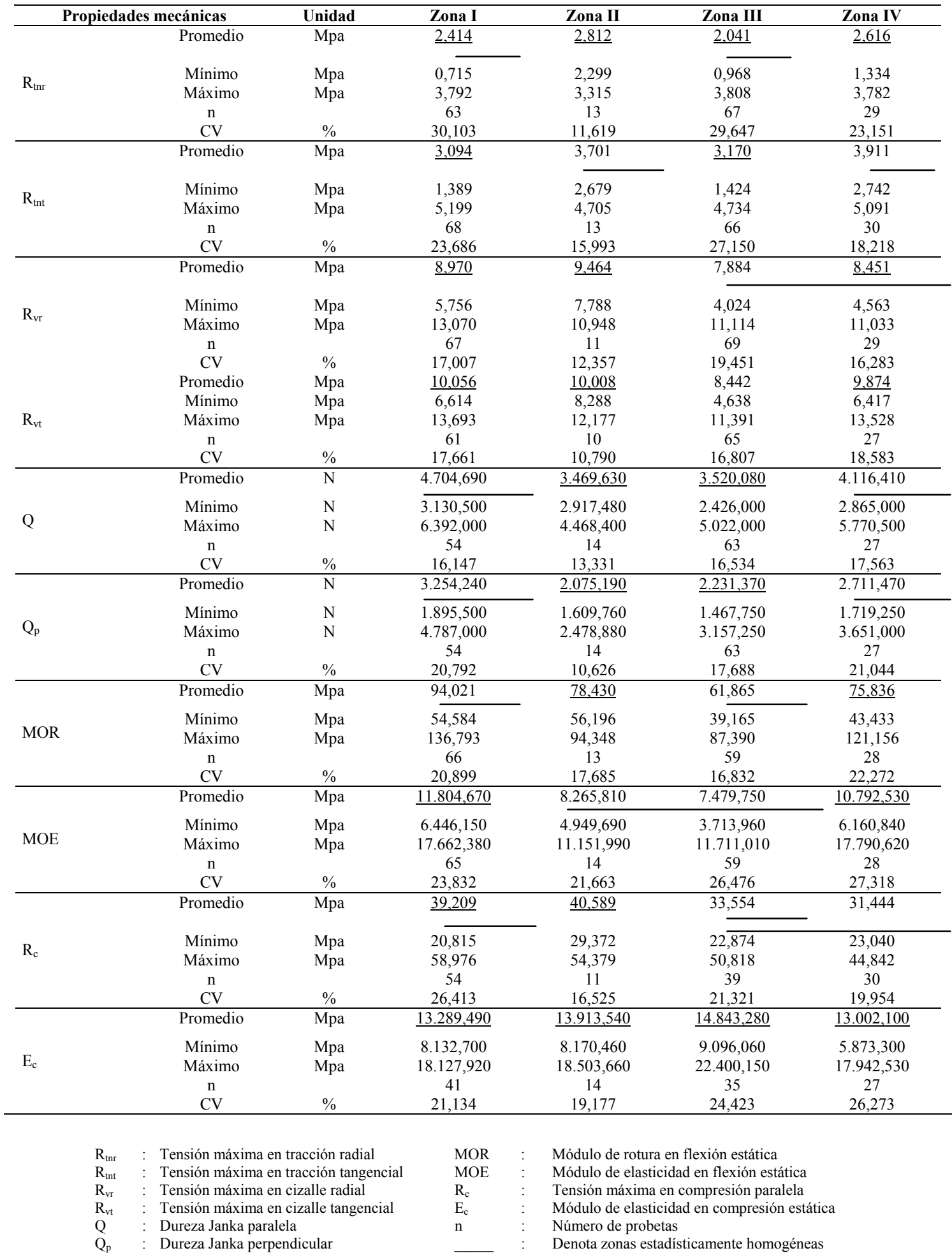

FLORESTA, Curitiba, PR, v. 37, n. 1, jan./abr. 2007.

Rozas, C.; Vargas, G.; Anzaldo, J. 
Tabla 3. Propiedades mecánicas en madera madura de Pinus radiata D. Don de 30 años, al $12 \%$ de contenido de humedad.

Table 3. Mechanical properties in adult Pinus radiata D. Don 30 year-old wood at 12\% moisture content.

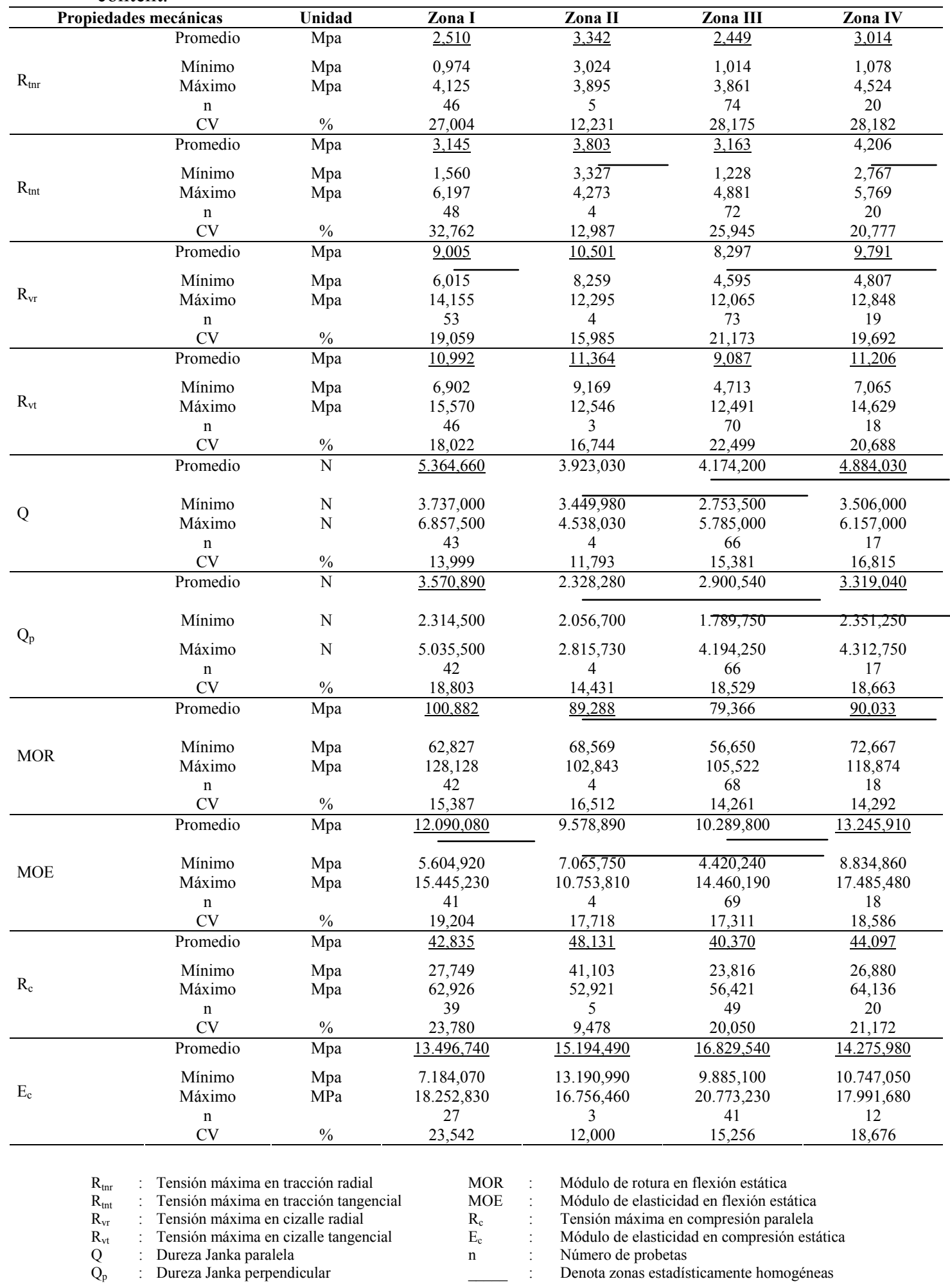


Entre las zonas I y IV, y entre las zonas II y III, se encontraron la mayor cantidad de propiedades mecánicas que no reflejaron diferencia estadística significativa. Entre las zonas I y II, se encontraron la mayor cantidad de propiedades que sí reflejaron diferencias estadísticas significativas; sin embargo, estas últimas representan el $20 \%$ del total de las propiedades estimadas.

\section{Modelos de regresión múltiple}

En la tabla 4 se muestran los modelos desarrollados para predecir las propiedades mecánicas en árboles de 30 años, provenientes de las cuatro zonas del centro-sur de Chile. Se puede observar, que todos los modelos señalados son significativos (valores-p $<0,01$ ) y donde el $50 \%$ de los modelos presentaron un $r^{2}$ superior a 0,50. El modelo que obtuvo un mayor coeficiente de determinación, fue $Q_{p}$ con $r^{2}=$ 0,8407 , mientras que el modelo que obtuvo el menor coeficiente de determinación, fue $\mathrm{R}_{\mathrm{tnr}}$ con $\mathrm{r}^{2}=$ 0,1528 .

Tabla 4. Modelos predictorios de las propiedades mecánicas de la madera de Pinus radiata D. Don de 30 años, al $12 \%$ de contenido de humedad.

Table 4. Mechanical properties models predictors from Pinus radiata D. Don 30 year-old wood at 12\% moisture content.

$$
\left(P, M,=\beta_{0}+\beta_{1} Z_{1}+\beta_{2} Z_{2}+\beta_{3} Z_{3}+\beta_{4} D+\beta_{5} M\right)
$$

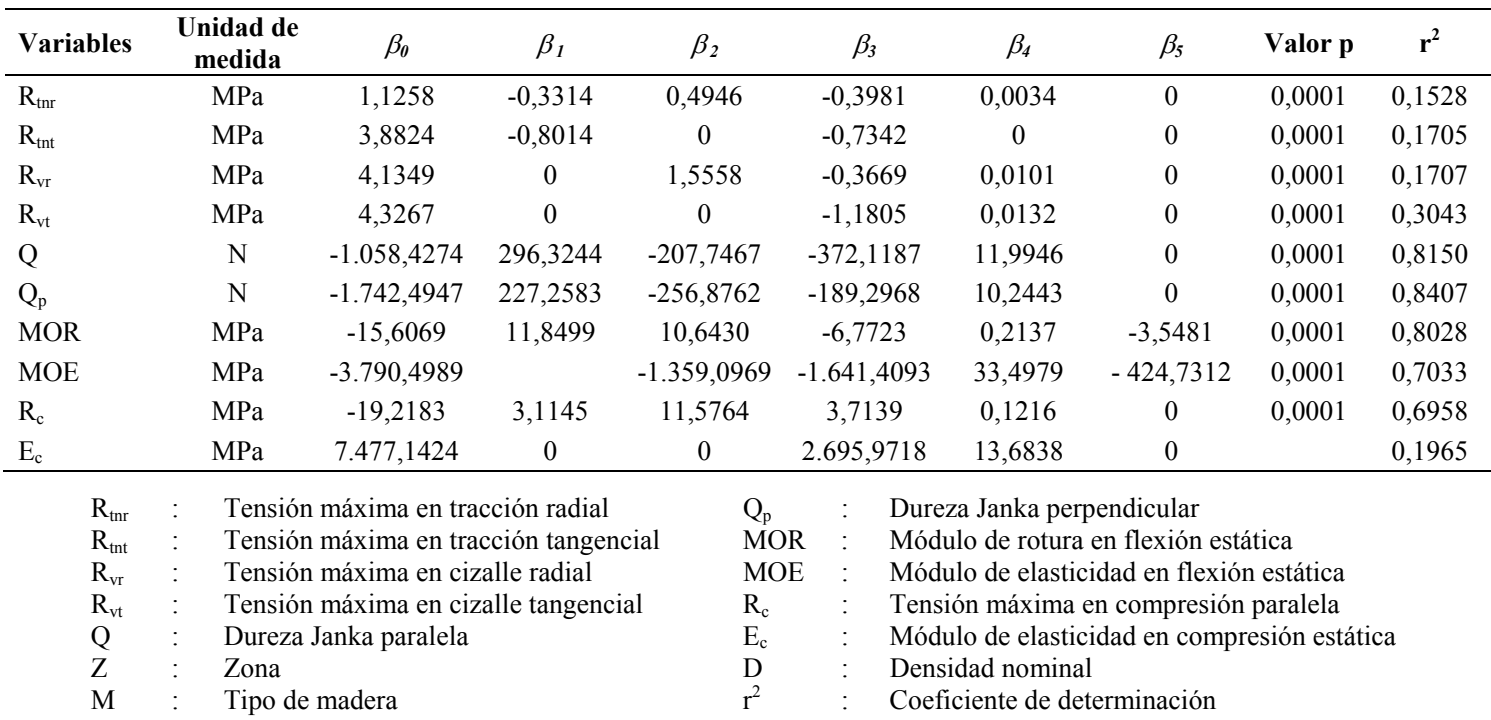

La variable densidad nominal fue significativa en todos los modelos, no así la variable tipo de madera (madera juvenil-madera adulta), que sólo fue significativa dentro del modelo para predecir el MOR y MOE (valor-p de su coeficiente superior a 10\%). Para este caso en particular, las variables densidad nominal y tipo de madera, explican más del $80 \%$ la capacidad predictiva de los modelos correspondientes.

Se puede observar además, por medio del coeficiente de determinación, que la tensión máxima en tracción radial y tangencial, no se correlacionan linealmente con la densidad y tipo de madera. Este comportamiento también fue observado en otro estudio similar realizado por Rozas et al. (2002), para árboles de 20 años de edad y en el estudio realizado por Juacida et al. (2000).

Los modelos anteriores incluyen las variables: zona (Z1, Z2, Z3 y Z4), tipo de madera (M) y densidad nominal (D), para las propiedades de tensión máxima en tracción radial $\left(\mathrm{R}_{\mathrm{tnr}}\right)$ y tangencial $\left(\mathrm{R}_{\mathrm{tnt}}\right)$, tensión máxima de cizalle radial $\left(R_{v r}\right)$ y tangencial $\left(R_{v t}\right)$, dureza paralela $(Q)$ y perpendicular $\left(Q_{p}\right)$, MOR y MOE en flexión, tensión máxima $\left(R_{c}\right)$ y módulo de elasticidad en compresión $\left(\mathrm{E}_{\mathrm{c}}\right)$. Para realizar estimaciones basadas en los modelos anteriores, se debe reemplazar la zona deseada por un "uno" (1) y las restantes por "cero" (0); excepto para las estimaciones de la zona IV (Z4), que en todos los casos se harán $Z_{\mathrm{i}}=0$. Para el caso tipo de madera, si se trata de madera juvenil, $M$ se debe reemplazar por "uno"

FLORESTA, Curitiba, PR, v. 37, n. 1, jan./abr. 2007. 
(1), y en el caso de madera adulta por "cero" (0). Por último se debe ingresar el valor de la densidad nominal en $\mathrm{kg} / \mathrm{m}^{3}$, en un rango de 308 a $585 \mathrm{~kg} / \mathrm{m}^{3}, 342$ a $476 \mathrm{~kg} / \mathrm{m}^{3}, 339$ a $497 \mathrm{~kg} / \mathrm{m}^{3}$ y 372 a $555 \mathrm{~kg} / \mathrm{m}^{3}$, válidos para los modelos de las zonas I, II, III y IV, respectivamente. La ausencia de algunas variables implica la baja influencia de éstas en las propiedades (valores-p superiores a 0,10 ).

\section{CONCLUSIONES}

De acuerdo a los resultados obtenidos se puede concluir:

- Para la madera juvenil, los mayores valores promedios se encontraron en árboles de 30 años de edad provenientes de la zona costera de Constitución - Cobquecura, seguidos de la zona de Concepción Angol, sur río Bío - Bío. El módulo de elasticidad en compresión paralela a las fibras, fue la única propiedad que no presentó diferencias estadísticas significativas entre las cuatro zonas.

- Para la madera adulta, los mayores valores medios de las propiedades mecánicas, se concentraron en la zona de Concepción - Angol, sur río Bío - Bío y en la zona costera de Constitución - Cobquecura. Generalmente, no se encontraron diferencias estadísticas significativas entre zonas, denotándose en el comportamiento de las propiedades de tensión máxima en cizalle tangencial, tensión máxima y módulo de elasticidad en compresión paralela a las fibras, que no presentaron diferencias entre las 4 zonas.

- Los modelos de regresión múltiple, indican que las predicciones de las propiedades mecánicas son satisfactorias a partir de la zona de procedencia, densidad nominal y tipo de madera, que permiten predecir las propiedades de dureza paralela, perpendicular, MOR, MOE y tensión máxima en compresión paralela. De estas propiedades la variable "densidad nominal" tiene un gran poder predictivo, y la variable "tipo de madera" sólo lo es para predicción del MOR y MOE, lo que indica estadísticamente que no es un buen predictor.

- En los modelos para estimar las propiedades de dureza paralela, perpendicular, MOR, MOE y tensión máxima en compresión paralela, que obtuvieron los mayores coeficientes de determinación $(0,8150$; 0,$8407 ; 0,8028 ; 0,7033$ y 0,6958 , respectivamente), las variables cualitativas "zonas", fueron altamente significativas (valores-p inferior al 10\%); a excepción del modelo para predecir el MOE.

- Los coeficientes de determinación de los modelos para predecir los esfuerzos máximos en tracción perpendicular y cizalle paralelo, y el módulo de elasticidad en compresión paralela a las fibras, indican la baja relación lineal con la densidad nominal, pese a que en su mayoría la densidad nominal fue significativa.

\section{REFERENCIAS}

ALBALA, H. Propiedades mecánicas y asociadas del pino insigne. In: Actas sobre investigación en productos forestales. Instituto Forestal, Santiago, Chile. n. 21, p. 59-67, 1964.

AMERICAN SOCIETY FOR TESTING AND MATERIALS. NORMA ASTM D 143-52: Standars methods of testing small clear specimens of timber. West Conshohocken, 1981.

BODIG, J.; JAYNE, B. Mechanics of wood and wood composites. Malabar: Krieger, 1993.

DÍAZ, M. 100 Años de presencia en Chile, Chile Forestal, Santiago de Chile, n.123, p. 12-13, 1985.

GALLARDO, R. Determinación de algunas propiedades mecánicas del Pinus radiata D. Don de la zona arenales Bulnes - Mulchén. Proyecto de Título, Ingeniería Civil en Industrias Forestales, Departamento de Ingeniería en Maderas, Universidad del Bío - Bío, Concepción, Chile, 1999.

INFOR. Estadísticas Forestales. Concepción, Chile, 1999.

INSTITUTO NACIONAL DE NORMALIZACIÓN. NCh 176/1: "Madera - Determinación de Humedad. Santiago, Chile, 1984a.

INSTITUTO NACIONAL DE NORMALIZACIÓN. NCh 176/2: Madera - Determinación de Densidad. Santiago, Chile, 1984a. 
INSTITUTO NACIONAL DE NORMALIZACIÓN. NCh 968: Madera - Selección, Obtención y acondicionamiento de muestras y Probetas para la Determinación de Propiedades Físicas y Mecánicas. Santiago, Chile, 1986a.

INSTITUTO NACIONAL DE NORMALIZACIÓN. NCh 969: Madera - Determinación de las Propiedades Mecánicas - Condiciones Generales para los Ensayos. Santiago, Chile, 1986 b.

INSTITUTO NACIONAL DE NORMALIZACIÓN. NCh 973: Madera - Determinación de las Propiedades Mecánicas - Ensayo de compresión paralela. Santiago, Chile, 1986c.

INSTITUTO NACIONAL DE NORMALIZACIÓN. NCh 975: Madera - Determinación de las propiedades mecánicas - Ensayo de tracción perpendicular a las fibras. Santiago, Chile, 1986d.

INSTITUTO NACIONAL DE NORMALIZACIÓN. NCh 976: Madera - Determinación de las propiedades mecánicas - Ensayo de cizalle paralelo a las fibras. Santiago, Chile, 1986e.

INSTITUTO NACIONAL DE NORMALIZACIÓN. NCh 978: Madera - Determinación de las propiedades mecánicas - Ensayo de dureza. Santiago, Chile, 1986f.

INSTITUTO NACIONAL DE NORMALIZACIÓN. NCh 987: Madera - Determinación de las propiedades mecánicas - Ensayo de flexión estática. Santiago, Chile, 1986g.

JOPIA, H. Modelamiento Estadístico para Determinar Algunas Propiedades Mecánicas de la Madera de Pinus radiata D. Don.. Proyecto de Título, Ingeniería Civil Industrial, Departamento de Ingeniería Civil Industrial, Universidad del Bío - Bío, Concepción, Chile, 2000.

JUACIDA, R.; CUEVAS, H.; SALDÍVAR, E. "Populus x euroamericana (Dode) Guinier: Aserrío, Secado y Propiedades Mecánicas”. Maderas: Ciencia y Tecnología, Concepción, Chile, v 2, n. 2, p. 185$195,2000$.

KARSUlOVIC, J. Propiedades mecánicas de la madera. Santiago, Chile: Facultad de Ciencias Agrarias, Veterinarias y Forestales, Universidad de Chile, 1982.

KININMONTH, J.; WhitehouSE, L. Properties and Uses of New Zealand Radiata Pine. Rotorua: Ministry of Forestry, Forest Research Institute, n. 8, p. 1-21, 1991.

PASTEN, R.; RUBILAR, J. Determinación de algunas propiedades mecánicas de la madera de Pinus radiata D. Don provenientes de la zona costera de Constitución - Cobquecura. Concepción, Chile: Universidad del Bío - Bío, 2001. Seminario de Título, Ingeniería de Ejecución en Maderas, Departamento de Ingeniería en Maderas.

PEREIRA, B. Determinación de propiedades mecánicas en Pinus radiata D. Don de 20 años. Concepción, Chile: Universidad del Bío - Bío, Concepción, Chile, 2000. Seminario de Título, Ingeniería de Ejecución en Maderas, Departamento de Ingeniería en Maderas.

PÉREZ, V.; CABRERA, J., Incidencia de la Edad del Árbol Sobre las Propiedades Físicas y Mecánicas del Pino radiata. Chile Forestal, Santiago de Chile, n.136, p. 26-28, 1987.

ROZAS, C.; PASTÉN, R. Caracterización de la madera de Pinus radiata D. Don, provenientes de la VII, VIII y IX región: Área Propiedades Mecánicas. Concepción, Chile: Universidad del Bío - Bío, 2001. Informes técnicos Proyecto FONDEF D97I2006. Departamento de Ingeniería en Maderas.

ROZAS, C.; PASTÉN, R.; VARGAS, G. Propiedades mecánicas en madera juvenil y madura de Pinus radiata D. Don, provenientes de la zona centro-sur de Chile. In: ENCONTRO BRASILEIRO EM MADEIRAS E EM ESTRUTURAS DE MADEIRA, 8., 2002,. Uberländia. Brasil. Caderno de resumos... Uberlândia: UFU, 2002. 